\title{
Psychrobacter isolates of human origin, other than Psychrobacter phenylpyruvicus, are predominantly Psychrobacter faecalis and Psychrobacter pulmonis, with emended description of $P$. faecalis
}

\author{
Pieter Deschaght, ${ }^{1}$ Michèle Janssens, ${ }^{2}$ Mario Vaneechoutte ${ }^{1}$ \\ and Georges Wauters ${ }^{2}$ \\ ${ }^{1}$ Laboratory Bacteriology Research (LBR), Department Clinical Chemistry, Microbiology, \\ Immunology, University of Ghent, Ghent, Belgium \\ ${ }^{2}$ Medical Microbiology, University of Louvain, UCL, Brussels, Belgium
}

Correspondence

Mario Vaneechoutte

Mario.Vaneechoutte@UGent.be
Human Psychrobacter isolates, other than Psychrobacter phenylpyruvicus, are predominantly designated Psychrobacter immobilis. Phenotypic and genotypic testing of Psychrobacter isolates that have been deposited in different culture collections as $P$. immobilis indicates that most of these human isolates belong to the species Psychrobacter faecalis and Psychrobacter pulmonis.
At present, all Psychrobacter isolates from human origin, other than Psychrobacter phenylpyruvicus (previously Moraxella phenylpyruvica), are designated Psychrobacter immobilis. Mostly, these are clinically not very relevant, but sometimes they are considered to be opportunistic pathogens (Gini, 1990; Lloyd-Puryear et al., 1991; Lozano et al., 1994). Many other Psychrobacter species have been described, mostly from the environment and from poikilothermic animals, but recently two species, Psychrobacter faecalis and Psychrobacter pulmonis, were isolated from warm-blooded animals, i.e. from pigeon faeces (Kämpfer

Abbreviations: CCUG, Culture Collection University of Göteborg; LMG, Laboratorium voor Microbiologie, Universiteit Gent; pNA, $p$-nitroanilide; pNP, $p$-nitrophenyl.

The GenBank/EMBL/DDBJ accession numbers for the 16S rRNA gene sequences reported in this paper are H0698589 (Psychrobacter immobilis ATCC 43116 $)$, HQ698586 (P. immobilis LMG 7085), HQ698587 ( $P$. nivimaris CCUG 39521), HQ698583 ( $P$. pulmonis LMG 7092), HO698585 (P. pulmonis UCL-NF 1589), HO698584 (P. pulmonis UCL-NF 1649), HO698582 (P. pulmonis LMG 1012), HQ698580 (P. pulmonis CCUG 41791), HQ698581 ( $P$. pulmonis CCUG 51808), H0698590 ( $P$. pulmonis UCL-NF 1791), HQ698579 ( $P$. pulmonis CCUG $\left.46240^{\top}\right)$, HO698562 ( $P$. faecalis DSM $\left.14664^{\top}\right)$, HO698578 ( $P$. faecalis UCL-NF 985), H0698564 ( $P$. faecalis LMG 7062), H0698569 ( $P$. faecalis LMG 6782), H0698588 ( $P$. faecalis UCL-NF 1590), H0698563 ( $P$. faecalis LMG 7060), HQ698565 ( $P$. faecalis LMG 7063), HQ698566 ( $P$. faecalis LMG 7061), H0698567 ( $P$. faecalis LMG 7059), HO698568 (P. faecalis LMG 5125), HO698570 ( $P$. faecalis LMG 7064), HQ698571 ( $P$. faecalis CCUG 45950), HQ698573 ( $P$. faecalis CCUG 52122), HQ698575 ( $P$. faecalis CCUG 55541), HQ698574 ( $P$. faecalis CCUG 54876), HQ698572 ( $P$. faecalis CCUG 51295), HQ698577 ( $P$. faecalis UCL-NF 322) and H0698576 ( $P$. faecalis CCUG 57827).

A supplementary table is available with the online version of this paper. et al., 2002) and from the lungs of a lamb (Vela et al., 2003), respectively. The type strain of $P$. immobilis, ATCC $43116^{\mathrm{T}}$, was isolated from poultry. Very recently, clinical isolates have been described as members of a novel species, Psychrobacter sanguinis (Wirth et al., 2012).

Since 2003, some human isolates have been deposited in the Culture Collection University of Göteborg (CCUG) collection and presumptively identified as $P$. faecalis or $P$. faecalislike. Because, to our knowledge, cases of human infection with $P$. faecalis have not yet been published and because none of the cases was caused by infection with P. immobilis, we compared our clinical isolates with the human isolates deposited in the CCUG and Laboratorium voor Microbiologie, Universiteit Gent (LMG) culture collections as $P$. immobilis and Psychrobacter sp. We examined a total of 26 human Psychrobacter isolates that did not belong to $P$. phenylpyruvicus present in two culture collections [CCUG $(n=9)$, LMG $(n=11)]$ and in the collection of the UCL (Georges Wauters, Brussels, Belgium; $n=6$ ) (Supplementary Table S1, available in IJSEM Online).

Determination of 16S rRNA gene sequences was carried out as described previously (Wauters et al., 2003) and this approach identified the following species: Psychrobacter cibarius ( $n=1$; LMG 7085), $P$. faecalis $(n=17)$, Psychrobacter nivimaris ( $n=1$; CCUG 39521) and P. pulmonis $(n=7)$ (Supplementary Table S1, Fig. 1). Cluster analysis of the 16S rRNA gene sequences (length $1456 \mathrm{bp}$ ) was performed using the Kodon software (Applied Maths, Sint-Martens-Latem, Belgium) according to the neighbour-joining method based on the multiple aligned distances algorithm (open gap cost, $100 \%$; unit gap cost, $20 \%$ ). A similar clustering result was 


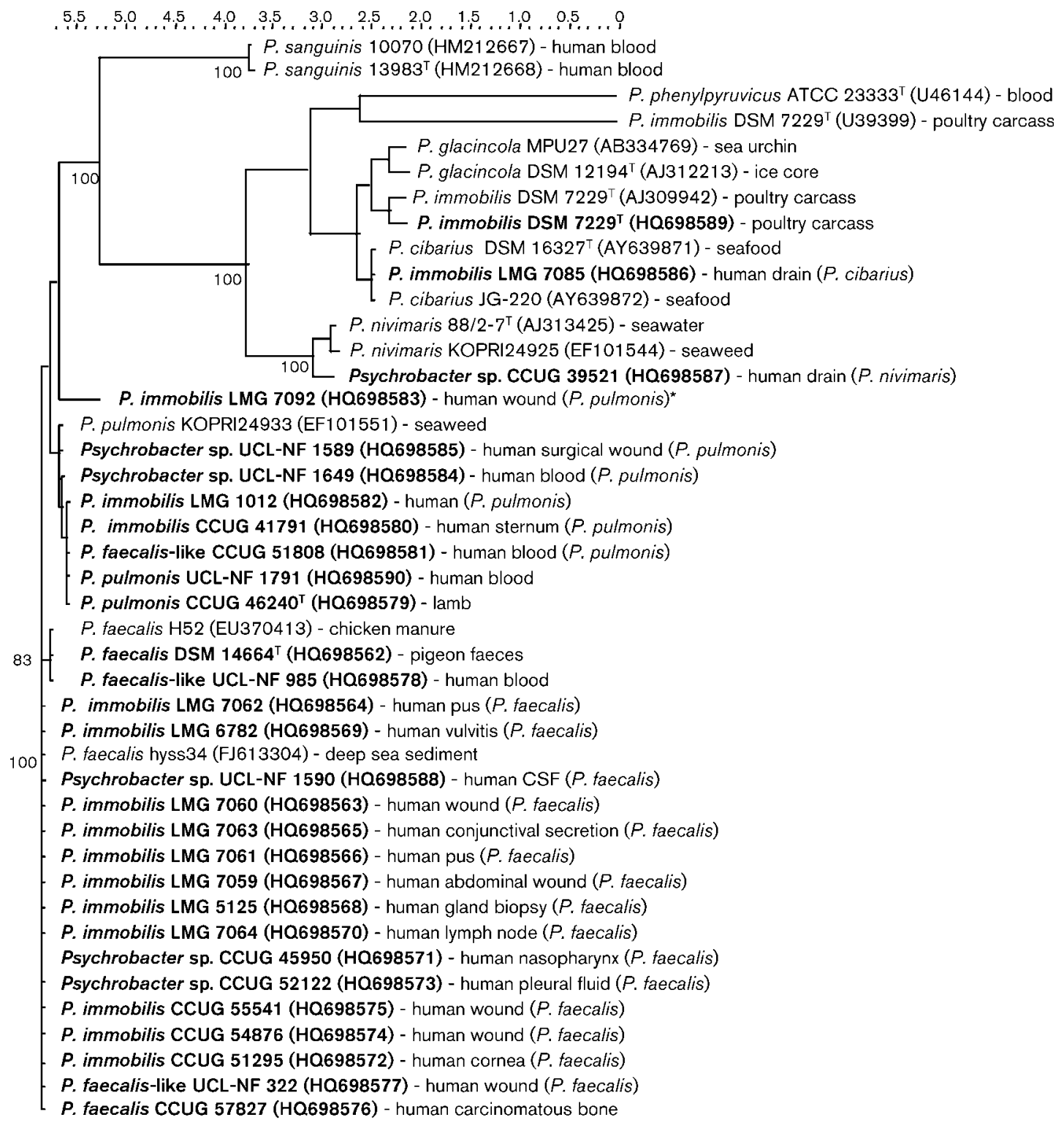

Fig. 1. Neighbour-joining phylogenetic tree based on 16S rRNA gene sequences of the Psychrobacter species included in this study. Bootstrap values greater than $80 \%$ are shown at the branch points. Sequences determined in this study are given in bold. Species names in parentheses are the final designations as determined in this study. *, Sequence is only $1291 \mathrm{bp}$ long, which probably explains its aberrant position.

obtained using the maximum-parsimony algorithm (with mutation cost $100 \%)$.

Three sequences have been deposited in GenBank for the type strain ATCC $43116^{\mathrm{T}}$ of P. immobilis, i.e. U39399 (Bowman et al., 1996), AJ309942 (Romanenko et al., 2002) and HQ698589 (this study). It should be noted that the sequence deposited under accession number U39399 differs at 33 positions from the same strain sequenced at our laboratory, whereas there are only six differences between our sequence and that deposited under accession number AJ309942.
Strains were grown on tryptic soy agar (TSA). Growth and biochemical tests were assessed at $30{ }^{\circ} \mathrm{C}$; growth was also determined at $35^{\circ} \mathrm{C}$. Oxidase activity was determined using dimethyl-p-phenylenediamine strips (Merck). Acid production from carbohydrates was detected on low-peptone phenol red (LPPR) agar (Wauters et al., 1998) and Hugh and Leifson's oxidation-fermentation medium (Hugh \& Leifson, 1953). For urease determination, liquid Christensen medium was used. Nitrate and nitrite reduction was detected using Griess reagent. A streak was inoculated onto TSA supplemented with $1 \%$ Tween 80 and $0.01 \% \mathrm{CaCl}_{2}$ for detection of Tween 80 esterase. For phenylalanine 
deaminase, phenylalanine agar was used. Paper discs containing $250 \mu \mathrm{g}$ desferrioxamine were used on MuellerHinton agar for susceptibility testing.

Phenotypic characterization of the Psychrobacter isolates indicated that all strains were positive for oxidase, catalase and tributyrin esterase, and susceptible to colistin, but negative for alkaline phosphatase, trypsin, pyrrolidonyl aminopeptidase, production of indole, $\beta$-galactosidase (ONPG), gelatin, aesculin hydrolase and arginine dihydrolase, and for growth at $42{ }^{\circ} \mathrm{C}$.

P. faecalis and P. pulmonis, although genotypically closely related ( $99 \%$ similarity between both type strains), as determined on the basis of $16 \mathrm{~S}$ rRNA gene sequences (Fig. 1), differed markedly in terms of their phenotypic characteristics. P. faecalis acidified a wide range of the carbohydrates listed in Table 1, and others not listed, i.e. lactose, galactose, melibiose, cellobiose and maltose (delayed), whereas $P$. pulmonis was asaccharolytic, as are all $P$. phenylpyruvicus isolates. Our finding of strong saccharolytic activity of $P$. faecalis on LPPR agar (Wauters et al., 1998) and Hugh and Leifson's oxidation-fermentation medium (Hugh \& Leifson, 1953) contradicts the original description of this species (Kämpfer et al., 2002), which states 'no acid production from carbohydrates'.

$P$. faecalis and $P$. pulmonis were urease-negative and nitrite reductase-positive, which easily differentiates them from

Table 1. Phenotypic characterization of Psychrobacter isolates of human origin, including type strains of $P$. faecalis, $P$. immobilis, $P$. phenylpyruvicus and $P$. pulmonis

Taxa: $1, P$. cibarius LMG $7085 ; 2$, $P$. faecalis $(n=18) ; 3$, P. immobilis ATCC $43116^{\mathrm{T}} ; 4$, P. nivimaris CCUG $39521 ; 5$, P. phenylpyruvicus $(n=7) ; 6$, P. pulmonis $(n=8)$.

\begin{tabular}{|lcccccc|}
\hline Characteristic & $\mathbf{1}$ & $\mathbf{2}^{\star}$ & $\mathbf{3}$ & $\mathbf{4}$ & $\mathbf{5}^{\star}$ & $\mathbf{6}^{*}$ \\
\hline Acid from glucose & + & + & + & + & - & - \\
Mannitol & - & - & - & - & - & - \\
Xylose & + & + & + & + & - & - \\
Arabinose & + & + & + & + & - & - \\
Ethylene glycol & + & $17 \dagger$ & + & + & 4 & + \\
Urease & + & - & + & - & + & - \\
Nitrate reductase & + & + & + & - & 2 & + \\
Nitrite reductase & - & + & - & - & - & 7 \\
Tween 80 hydrolase & + & $17 \dagger$ & + & + & + & + \\
Phenylalanine & - & 1 & - & - & 6 & - \\
$\quad$ deaminase & & & & & & \\
Desferrioxamine & - & $1 \ddagger$ & - & - & - & 2 \\
$\quad$ susceptibility & & & & & & \\
Growth at $35{ }^{\circ} \mathrm{C}$ & - & + & + & - & + & + \\
\hline
\end{tabular}

${ }^{\star}$ Where result is not the same for all strains studied, the number of positive strains is indicated.

†Of the 18 strains studied, only UCL-NF 1590 is negative.

$\ddagger$ Type strain is positive.
P. phenylpyruvicus and $P$. immobilis. Furthermore, nitrite reductase was only positive in these two species (except for one $P$. pulmonis strain), in contrast to all other strains tested in this study. Almost all Psychrobacter species were positive for Tween hydrolysis; it is of interest to note that growth of $P$. phenylpyruvicus is drastically enhanced by addition of $1 \%$ Tween 80 to the medium. Strain UCL-NF 1590 , a genuine $P$. faecalis strain according to its $16 \mathrm{~S}$ rRNA gene sequence, is the only isolate that is unable to hydrolyse Tween 80 or acidify ethylene glycol in this species.

Apart from the saccharolytic properties of $P$. faecalis, we found no discrepancies with the results already described for this species and the other species tested here.

We conclude that most human Psychrobacter isolates of European origin, other than $P$. phenylpyruvicus, belong predominantly to the species $P$. faecalis and $P$. pulmonis, and not to $P$. immobilis. We propose that the description of $P$. faecalis is emended to include the strong saccharolytic activity observed in all the $P$. faecalis strains tested in this study.

\section{Emended description of Psychrobacter faecalis}

Psychrobacter faecalis (fae.ca'lis. L. n. faex faecis dregs; L. masc. suff. -alis suffix denoting pertaining to; N.L. masc. adj. faecalis fecal).

This description is based on that of Kämpfer et al. (2002). Unless marked otherwise, most data are from this study. However, chemotaxonomic data and assimilation/utilization test data are from Kämpfer et al. (2002) and are based on the type strain only, which was the only strain available at the time of the original description. Cells are straight rods, $0.8-$ $1.2 \times 1.0-2.0 \mu \mathrm{m}$. Cells occur singly and are non-motile, Gram-negative, oxidase-positive and catalase-positive, with an oxidative, chemoheterotrophic metabolism. Ubiquinone Q-8 is the major quinone type, with low amounts of ubiquinone Q-9 and Q-7; spermidine is the major polyamine. The polar lipid profile is characterized by the presence of phosphatidylethanolamine, phosphatidylglycerol and diphosphatidylglycerol. The fatty acids $\mathrm{C}_{18: 1} \omega 9 c, \mathrm{C}_{17: 1} \omega 8 c$ and summed feature $3\left(\mathrm{C}_{16: 1} \omega 7 c\right.$ and/or iso- $\left.\mathrm{C}_{15: 0} 2-\mathrm{OH}\right)$, in addition to the hydroxylated fatty acid $\mathrm{C}_{12: 0} 3-\mathrm{OH}$, are produced. On nutrient agar, colonies are circular, opaque, slightly raised and beige with entire margins. The type strain Iso- $46^{\mathrm{T}}$ grows well at $4-36{ }^{\circ} \mathrm{C}$ on different complex media, such as R2A agar and nutrient agar. No growth is observed at 45 or $55{ }^{\circ} \mathrm{C}$ on nutrient agar. No growth occurs on Salmonella/Shigella agar. $\mathrm{H}_{2} \mathrm{~S}$ is not produced. Negative for indole production, urease, arginine dihydrolase, lysine decarboxylase, ornithine decarboxylase and growth on Simmons' citrate. Saccharolytic with acid production from glucose, arabinose and xylose but not from mannitol. Acid is also produced from ethylene glycol. Utilization of $\mathrm{N}$-acetyl-Dgalactosamine, $\mathrm{N}$-acetyl-D-glucosamine, L-arabinose (weak), D-glucose (weak), acetate, propionate (weak), cis-aconitate, 4-aminobutyrate, citrate, fumarate, glutarate, DL-3-hydroxybutyrate, DL-lactate, L-malate, 2-oxoglutarate, pyruvate, L-alanine, L-aspartate, L-leucine and L-ornithine (weak) is 
positive. The following compounds are not utilized as sole carbon sources: L-arbutin, cellobiose, D-galactose, gluconate, maltose, D-mannose, $\alpha$-melibiose, L-rhamnose, D-ribose, sucrose, salicin, trehalose, D-xylose, adonitol, $i$-inositol, Dsorbitol, glycerol, D-fructose, D-mannitol, maltitol, putrescine, trans-aconitate, mesaconate, adipate, azelate, itaconate, suberate, $\beta$-alanine, L-phenylalanine, L-serine, L-proline, L-histidine, L-tryptophan, 3-hydroxybenzoate, 4-hydroxybenzoate and phenylacetate. The chromogenic substrates $p$-nitrophenyl (pNP)- $\alpha$-D-glucopyranoside, $\mathrm{pNP}-\beta$-D-galactopyranoside, ONPG, $\mathrm{pNP}-\beta$-D-glucuronide, $\mathrm{pNP}-\beta$-D-glucopyranoside, pNP- $\beta$-D-xylopyranoside, 2 -deoxythymidine$5^{\prime}$-pNP-phosphate, L-glutamate- $\gamma$-3-carboxy- $p$-nitroanilide (pNA), L-proline-pNA and pNP-phosphorylcholine are not hydrolysed. Only hydrolysis of L-alanine-pNA and bis-pNPphosphate is positive.

The type strain is Iso- $46^{\mathrm{T}}\left(=\mathrm{DSM} 14664^{\mathrm{T}}=\mathrm{CIP} 107288^{\mathrm{T}}\right)$, isolated on MacConkey agar from a bioaerosol originating from pigeon faeces.

\section{References}

Bowman, J. P., Cavanagh, J., Austin, J. J. \& Sanderson, K. (1996). Novel Psychrobacter species from Antarctic ornithogenic soils. Int $J$ Syst Bacteriol 46, 841-848.

Gini, G. A. (1990). Ocular infection caused by Psychrobacter immobilis acquired in the hospital. J Clin Microbiol 28, 400-401.

Hugh, R. \& Leifson, E. (1953). The taxonomic significance of fermentative versus oxidative metabolism of carbohydrates by various Gram negative bacteria. J Bacteriol 66, 24-26.
Kämpfer, P., Albrecht, A., Buczolits, S. \& Busse, H. J. (2002). Psychrobacter faecalis sp. nov., a new species from a bioaerosol originating from pigeon faeces. Syst Appl Microbiol 25, 31-36.

Lloyd-Puryear, M., Wallace, D., Baldwin, T. \& Hollis, D. G. (1991). Meningitis caused by Psychrobacter immobilis in an infant. J Clin Microbiol 29, 2041-2042.

Lozano, F., Florez, C., Recio, F. J., Gamboa, F., Gómez-Mateas, J. M. \& Martín, E. (1994). Fatal Psychrobacter immobilis infection in a patient with AIDS. AIDS 8, 1189-1190.

Romanenko, L. A., Schumann, P., Rohde, M., Lysenko, A. M., Mikhailov, V. V. \& Stackebrandt, E. (2002). Psychrobacter submarinus sp. nov. and Psychrobacter marincola sp. nov., psychrophilic halophiles from marine environments. Int J Syst Evol Microbiol 52, 1291-1297.

Vela, A. I., Collins, M. D., Latre, M. V., Mateos, A., Moreno, M. A., Hutson, R., Domínguez, L. \& Fernández-Garayzábal, J. F. (2003). Psychrobacter pulmonis sp. nov., isolated from the lungs of lambs. Int $\mathrm{J}$ Syst Evol Microbiol 53, 415-419.

Wauters, G., Van Bosterhaut, B., Janssens, M. \& Verhaegen, J. (1998). Identification of Corynebacterium amycolatum and other nonlipophilic fermentative corynebacteria of human origin. J Clin Microbiol 36, 1430-1432.

Wauters, G., Avesani, V., Laffineur, K., Charlier, J., Janssens, M., Van Bosterhaut, B. \& Delmée, M. (2003). Brevibacterium lutescens sp. nov., from human and environmental samples. Int J Syst Evol Microbiol 53, 1321-1325.

Wirth, S. E., Ayala-Del-Río, H. L., Cole, J. A., Kohlerschmidt, D. J., Musser, K. A., Sepúlveda-Torres, Ldel. C., Thompson, L. M. \& Wolfgang, W. J. (2012). Psychrobacter sanguinis sp. nov., recovered from four clinical specimens over a 4-year period. Int J Syst Evol Microbiol 62, 49-54. 